\title{
Surgical Challenges in a Case of a Recurrent Giant Pleomorphic Liposarcoma of Neck And Mediastinum in a Child: A Rare Occurrence
}

\author{
Anand Kumar Mishra ${ }^{1}$ - Ankit Singhal ${ }^{1} \cdot$ Ramya Rathod $^{2} \cdot \operatorname{Vidur}^{\text {Bansal }}{ }^{1}$ (D) \\ Naresh Kumar Panda ${ }^{2} \cdot$ Sunder Negi $^{3} \cdot$ Pratyaksha Rana $^{4}$
}

Received: 20 June 2021/Accepted: 21 August 2021/Published online: 27 August 2021

(C) Association of Otolaryngologists of India 2021

\begin{abstract}
Liposarcomas are rare in childhood, representing $<1 \%$ of all childhood soft tissue sarcomas. The most frequent site of involvement are the extremities with the involvement of head and neck being an absolute rarity. Pleomorphic subtype of liposarcoma is pretty aggressive and hence portends a poor prognosis. Here, we present the case of a child who presented with a recurrence of liposarcoma in the neck and mediastinum within 3 months of the index surgery.
\end{abstract}

Keywords Liposarcoma - Recurrence - Cervical · Mediastinum

\section{Introduction}

Liposarcomas are tumors derived from primitive mesenchymal cells that undergo adipose differentiation. Liposarcomas are predominantly a disease of adulthood with sparse reports in childhood [1]. Primary cervical or mediastinal liposarcomas are rare. They are often

Vidur Bansal

vidurbansal05@gmail.com

1 Department of Cardiothoracic and Vascular Surgery, Post Graduate Institute of Medical Education and Research, Chandigarh, India

2 Department of Otolaryngology, Post Graduate Institute of Medical Education and Research, Chandigarh, India

3 Department of Anesthesia, Post Graduate Institute of Medical Education and Research, Chandigarh, India

4 Department of Radiodiagnosis and Imaging, Post Graduate Institute of Medical Education and Research, Chandigarh, India asymptomatic and usually present with compression symptoms related to direct invasion or compression of other thoracic organs such as the heart, great vessels or the airway. Liposarcomas are rare in the pediatric population and involvement of the neck is even rarer, hence, the optimal management is still unknown. But like any other malignancy, wide local excision with negative margins followed by adjuvant chemo-radiotherapy is recommended. To the best of our knowledge, this is the first report of a child with such a massive recurrence in the neck extending into the mediastinum.

\section{Case Report}

A 10 year old girl underwent a right hemithyroidectomy in May, 2020 for a right sided thyroid swelling. The lesion was found to be continuing into the retrosternal and tracheoesophageal groove intra-operatively. Histopathology was suggestive of pleomorphic liposarcoma. Soon after discharge from the hospital, her parents began to notice a swelling in front of the neck which gradually increased in size. After initial evaluation at the same hospital, she was diagnosed to have a recurrence in August, 2020 and was started on chemotherapy (Adriamycin and Ifosfamide). The ongoing pandemic probably further delayed the treatment, so much so that she completed 6 cycles in February, 2021. But, still her symptoms worsened and she was referred to our hospital due to lack of symptomatic improvement and progressive increase in the size of the swelling. The child became more dyspneic and even complained of orthopnea by the time she was referred to our hospital. The child was planned for radiotherapy first, but as the child became more symptomatic after 1 cycle of radiotherapy mostly due to the underlying tissue edema, it was decided to discontinue 
further radiotherapy and proceed with emergency surgery. She also had a strong family history of malignancy on the maternal side and was subsequently found out to be heterozygous for Li-Fraumeni syndrome. On local examination, there was a $11 \times 6 \mathrm{~cm}$ multilobulated mobile swelling involving the central part of neck and the inferior border was not palpable because of the retrosternal extension. The latest computed tomography(CT) scan (Fig. 1) revealed a large lobulated hypodense lesion involving the neck and mediastinum causing severe narrowing of the trachea $(2.2 \mathrm{~mm}$ in maximum expiration) without any infiltration. The mass completely encased superior vena cava (SVC), Right brachiocephalic vein (BCV), Innominate artery, left and right common carotid artery (CCA) with normal contrast opacification (Fig. 2). It partially encased right pulmonary artery (RPA) and was seen compressing the right atrium without infiltration. On indirect laryngoscopy, she had right vocal cord palsy. She subsequently underwent a positron emission tomography (PET) scan, which revealed similar findings and no distant metastasis. The child also underwent transthoracic echocardiography which revealed a large mediastinal mass overlying the right atrium, right ventricle and partially over the main pulmonary artery without any inflow or outflow obstruction. Keeping all this in mind, the patient was intubated while spontaneously ventilating under bronchoscopic guidance. An armoured endotracheal tube of size $4.5 \mathrm{~mm}$ was initially passed with ease which was later exchanged to a bigger tube of $5.5 \mathrm{~mm}$. Through an initial collar incision and with rotation of the carotid sheaths laterally, a well encapsulated $11 \times 4 \mathrm{~cm}$ mass was dissected from behind the hypopharynx. As it extended far into the mediastinum, a sternotomy was performed and bilateral pleural cavities were opened which helped in mobilization and precise dissection. We went ahead with the sternotomy from below upwards using a heavy Mayo scissors rather than the oscillating saw which could have cut through the tumour. Anticipating difficulty in intubation owing to severe narrowing of the trachea, a cardiopulmonary bypass (CPB) machine was kept as a standby where we planned to cannulate the femoral vessels and initiate CPB but fortunately we could do it without CPB. The liposarcoma surrounded the aortic arch, and was lying anterior and lateral to the trachea. First, we proceeded with

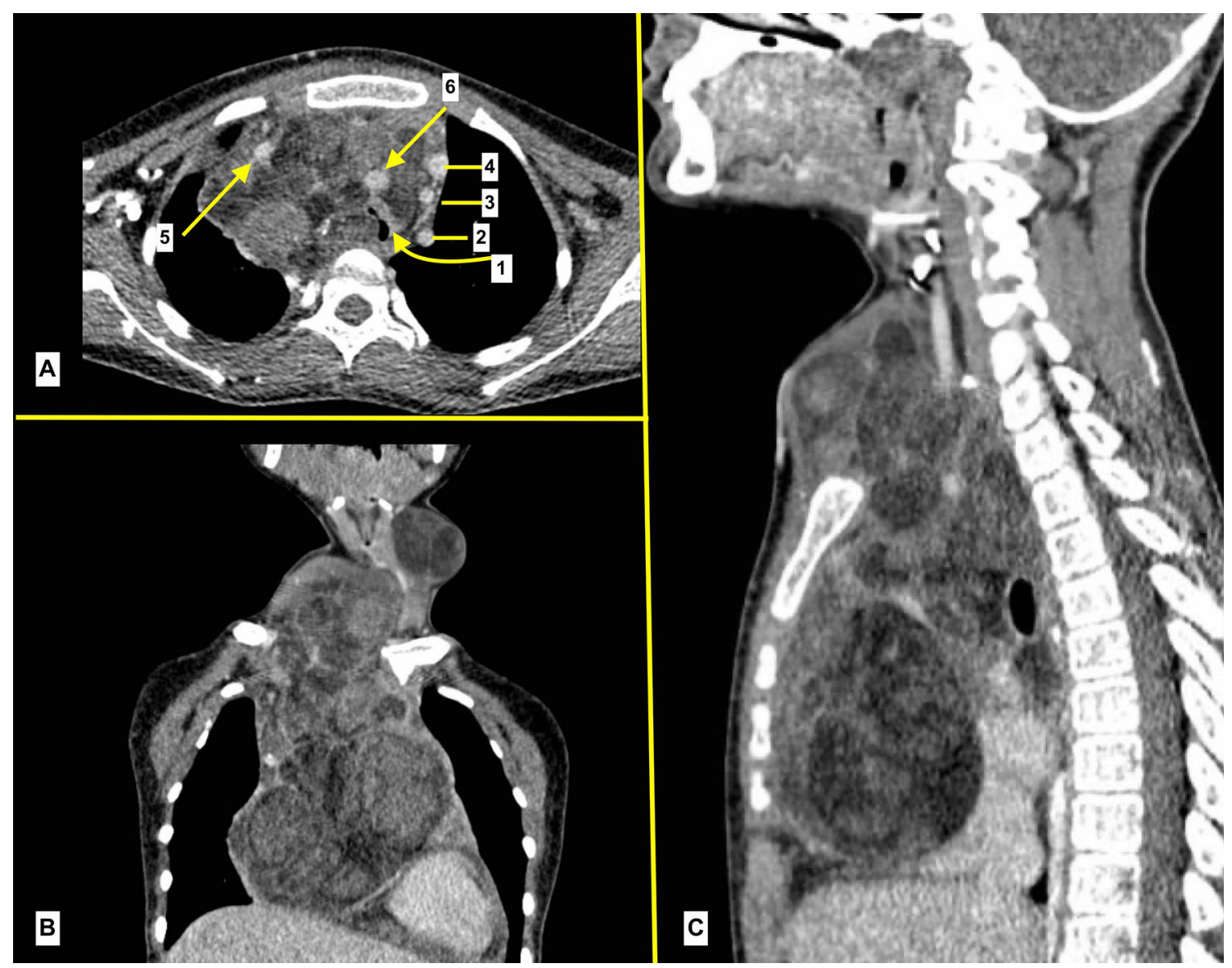

Fig. 1 CT scan (May-2021) showing the extensive spread of the tumour, A Axial section, B Coronal Section, C Sagittal section; 1-Tracheal lumen severely compromised, 2-Left SCA, 3-Left CCA, 4-Left Brachiocephalic vein, 5-Right Brachiocephalic vein, 6-Brachiocephalic artery 


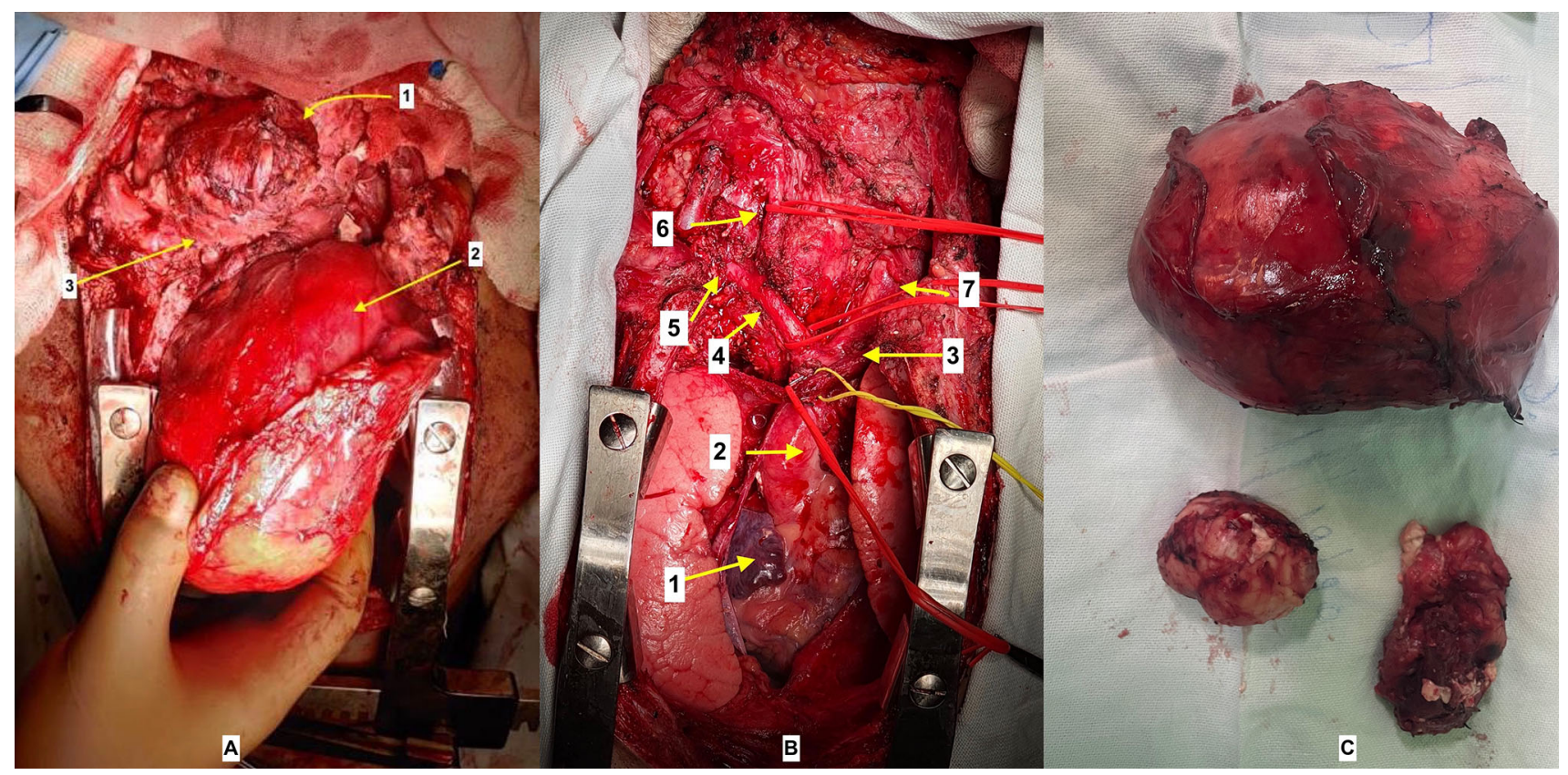

Fig. 2 Intra-operative Images-A After dissection of the tumour, 1-Cervical part of the tumour, 2-Mediastinal part of the tumour, 3-Left Innominate vein, B After removal of the tumour, 1-Right Atrium, 2-Ascending aorta, 3-Left Innominate vein, 4-Innominate

the dissection in the neck followed by dissection over the right atrium (RA) and right ventricle (RV), followed by dissection over the innominate vein and arch vessels. The tumor was dissected from under the brachiocephalic artery and rotated down from the neck. Since, the lesion was too large, lobulated portions of the mass were removed. A final component was dissected off the distal arch of the aorta to complete the resection. A small part of the capsule of the tumour was left behind near the junction of the SVC and BCV since it was densely adherent to it. The child was tracheostomized on table because her right vocal cord was already damages and although we identified and preserved the left recurrent laryngeal nerve, we were sceptical of its damage. Postoperatively the patient was gradually weaned off ventilator support and was discharged to home on POD8. Histopathology revealed pleomorphic liposarcoma. The child is doing well in the postoperative period.

\section{Discussion}

In our case, surgical planning and a multidisciplinary team approach were very important for the outcome of the child. The tumour in this case, was completely encasing the aortic arch and its branches, which posed an important question artery, 5-Right subclavian artery, 6-Right Common carotid artery, 7-Left common carotid artery, C Excised specimen

before us about the resectability of the tumour. On further discussions, intubating the child was a major hurdle. Also, on preoperative imaging, the tumour was so huge, planning a sternotomy was very important. We made all necessary arrangement to put the patient on CPB as soon as the need arose to do so. We could excise the tumour without use of a CPB machine which had important implications. Postoperatively, we have planned to administer both chemotherapy and radiotherapy to this child and keep her under close surveillance.

Liposarcomas are very rare in children [2]. Pathologically four main types of liposarcomas have been described: myxoid, well differentiated, dedifferentiated and pleomorphic [3]. One of the most widely used grading systems for soft tissue sarcomas, the French Federation of Cancer Centers Sarcoma Group systems uses a 3-tier grading system to separate tumors based on based on mitotic activity, necrosis, and differentiation [4]

Surgical decision-making gets problematic in locally advanced tumors when neoadjuvant treatment modalities fail, and an R0 resection requires a drastic functional impairment. Local control should be achieved by radiation, chemotherapy, or surgical debulking, as recommended by the current National Comprehensive Cancer Network guidelines [5, 6]. 


\section{Conclusion}

Pleomorphic liposarcomas of the head and neck are exceedingly rare tumors with a poor prognosis. Surgical removal is the optimal treatment for a cervico-mediastinal liposarcoma. If the entire tumor cannot be resected, surgical debulking often results in symptomatic relief. International literature has demonstrated that recurrent disease occurs and therefore a long-term careful follow up is required. Since liposarcoma is uncommon in childhood and may differ from the same disease in adults, a large randomized trial is required to formulate a definitive plan for optimal management.

Author Contributions VB-Data analysis, Manuscript writing, AS-Data collection, Conceptualization, RR-Manuscript writing, Data collection, AKM-Manuscript editing, Data analysis, NKPData collection, Manuscript editing, SN-Manuscript editing, Data analysis, PR-Manuscript writing, Data collection.

Funding No funds were received for this work. No prior abstract or poster presentation for this case.

Data Availability Yes.

\section{Declarations}

Conflict of interest The author declare no conflict of interest.
Ethical Approval This manuscript was approved by the departmental ethics committee.

Informed Consent Informed consent was taken from the child's parents for publication purposes.

\section{References}

1. Huh WW, Yuen C, Munsell M, Hayes-Jordan A, Lazar AJ, Patel S et al (2011) Liposarcoma in children and young adults: a multiinstitutional experience. Pediatr Blood Canc 57:1142-1146

2. Gerry D, Fox NF, Spruill LS, Lentsch EJ (2014) Liposarcoma of the head and neck: analysis of 318 cases with comparison to nonhead and neck sites. Head Neck 36:393-400

3. Fletcher CDM, Hogendoorn PCW, Mertens F et al (2013) WHO Classification of Tumours of Soft Tissue and Bone. DC, IARC Press, Washington

4. Guillou L, Coindre JM, Bonichon F et al (1997) Comparative study of the national cancer institute and french federation of cancer centers sarcoma group grading systems in a population of 410 adult patients with soft tissue sarcoma. J Clin Oncol 15:350-362

5. von Mehren M, Randall RL, Benjamin RS, et al. Soft tissue sarcoma, version 2.2016, NCCN Clinical Practice Guidelines in Oncology. J Natl Compr Canc Netw 2016;14:758-86

6. Munden RF, Nesbitt JC, Kemp BL, Chasen MH, Whitman GJ (2000) Primary liposarcoma of the mediastinum. AJR 175:1340

Publisher's Note Springer Nature remains neutral with regard to jurisdictional claims in published maps and institutional affiliations. 